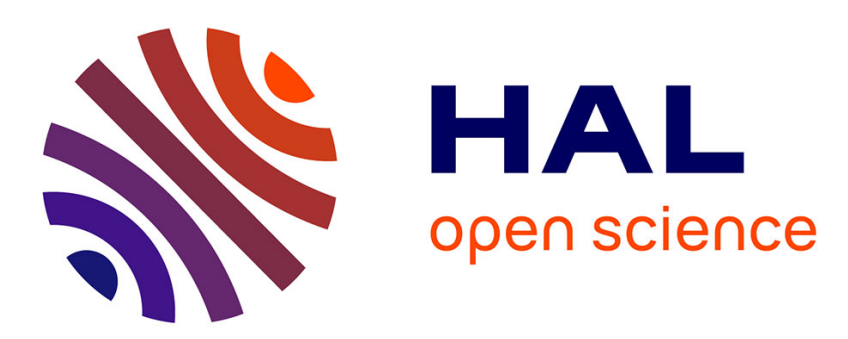

\title{
Fully automatic analysis of archival aerial images current status and challenges
}

Sébastien Giordano, Arnaud Le Bris, Clément Mallet

\section{To cite this version:}

Sébastien Giordano, Arnaud Le Bris, Clément Mallet. Fully automatic analysis of archival aerial images current status and challenges. 2017 Joint Urban Remote Sensing Event (JURSE), Mar 2017, Dubai, United Arab Emirates. pp.1-4, 10.1109/JURSE.2017.7924620 . hal-02386684

\section{HAL Id: hal-02386684 https://hal.science/hal-02386684}

Submitted on 29 Nov 2019

HAL is a multi-disciplinary open access archive for the deposit and dissemination of scientific research documents, whether they are published or not. The documents may come from teaching and research institutions in France or abroad, or from public or private research centers.
L'archive ouverte pluridisciplinaire HAL, est destinée au dépôt et à la diffusion de documents scientifiques de niveau recherche, publiés ou non, émanant des établissements d'enseignement et de recherche français ou étrangers, des laboratoires publics ou privés. 


\title{
Fully Automatic Analysis of Archival Aerial Images Current status and challenges
}

\author{
Sébastien Giordano, Arnaud Le Bris, Clément Mallet \\ IGN-ENSG, LaSTIG-MATIS - Univ. Paris-Est, FRANCE \\ \{firstname. lastname\}@ign.fr
}

\begin{abstract}
Archival aerial images are a unique and relatively unexplored means to generate detailed land-cover information in 3D over the past 100 years. Many long-term environmental monitoring studies can be based on this type of image series. Such data provide a relatively dense temporal sampling of the territories with very high spatial resolution. Furthermore, photogrammetric workflows exist in order to both produce orthoimages and Digital Surface Models, with reasonable interactive actions. However, today, there is no fully automatic pipeline for generating such kind of data. This paper presents the main avenues of research in order to develop such workflow, starting from registration and radiometric issues up to land-cover classification challenges.
\end{abstract}

Keywords-archival aerial images, time series analysis, photogrammetry, registration, classification.

\section{INTRODUCTION}

Understanding the $20^{\text {th }}$ century dynamics of urban environments is an invaluable asset: sustainable development indicators can be derived and impacts of land urban planning policies can be simulated.

Until now, satellite images and archival topographic maps have been used to characterise urban dynamics. On the one hand, satellite images are compatible with automatic analysis criteria, as their radiometry and spatial resolution are stable over time. For instance, Landsat images have been widely used in order to monitor urban land cover change [1]. However, the type of dynamics that can be described are limited by the spatial resolution $(30-60 \mathrm{~m})$, the time interval of the time series (30-40 years), and to $2 \mathrm{D}$. On the other hand, archival topographic maps are available in many countries since the $18^{\text {th }}$ century, but are difficult to analyse automatically. Thus, approaches that characterise urban evolution are heavily based on Volunteered Geographic Information [2]. Moreover, their temporal resolution is very coarse: the objects that can be extracted from the maps difficult to date precisely [3], [4].

Another source of information for effective long-term environmental monitoring and change detection is archival aerial images. They offer an unprecedented insight into the past [5]. There characteristics are perfectly tailored for urban area analysis. First, images have regularly been acquired from the 1920 's by defence, national mapping, or cadastral agencies, often with vertical camera axis and stereoscopic configuration, in order to produce or update topographic maps. Secondly, they offer a very high spatial resolution $(<1-2 \mathrm{~m})$, that allows (i) generating precise Digital Surface Models (DSMs), and (ii) discriminating a potential large number of urban classes. However, such advantages are accompanied with very particular characteristics. They are usually composed of an unique panchromatic channel, and their radiometry can be very variable. The digital images are generated from old analog photography, acquired with different cameras for which only coarse image localisation was stored. In addition, artefacts and noise are often observed. As a result, radiometric issues heavily impact the geometric accuracy of the orthoimages [5], and subsequently of the DSMs. Moreover, analysing these time series is rather challenging since images exhibit highly heterogeneous spatial resolutions, with very different acquisition conditions. In order to identify the needs for new solutions, this paper presents the main challenges so as to automatically analyse such type of images.

\section{CURRENT STATUS}

The growing initiatives to facilitate the access to archival aerial images, the availability of digital photogrammetric/dense matching softwares, and the numerous applications that can be based on historical images, make it timely to start using these images for chronicling and studying land-cover dynamics.

\section{A. Increasing availability of archival images}

General statements: Performing aerial surveys in order to produce topographic maps has been a common practice in many countries over the last century. Recently, several countries have decided to digitise their archival film-based photos, and to facilitate their free access through dedicated spatial data infrastructures and web services [6], [7]. Some companies have also started developing services to process archival images. Alternatively, scanning projects are steadily increasing in number. Useful metadata is generally distributed in addition to the images, such as length and width of the analog photos, focal length of the camera, and more rarely camera calibration reports. As a consequence, data available for end-users is consistent with standard photogrammetric processing chains.

Heterogeneous datasets: Existing analog and digital images available over an area of interest are bound to exhibit highly heterogeneous specifications over a very large range of time: spatial resolutions, spectral channels, survey month, etc. A study area of $3 \times 2 \mathrm{~km}$ located in the South of France (cities of Fréjus Saint-Raphaël) has been selected to illustrate these facts, and for further processing. 50 aerial surveys from 1926 to 2003 are available, showing a relatively dense temporal sampling of urban areas. In Figure 1, one may note that: 
- There is a clear and obvious trend, moving from panchromatic to natural images (red, green, blue channels), with punctually other spectral channels (infrared channel);

- The spatial resolution and the season vary a lot. This is explained by the fact that no standard national survey procedure was set up in France before the 1990's: each survey was tailored for a specific need. This represents a realistic situation that other countries are likely to face.

- These archival datasets are consistent with many applications dealing with the evolution of urban areas: e.g., images every 5 years, spatial resolution of less than $1 \mathrm{~m}$.

Aerial surveys were carried out for map generation or update: stereoscopic configuration was available for almost all dates.

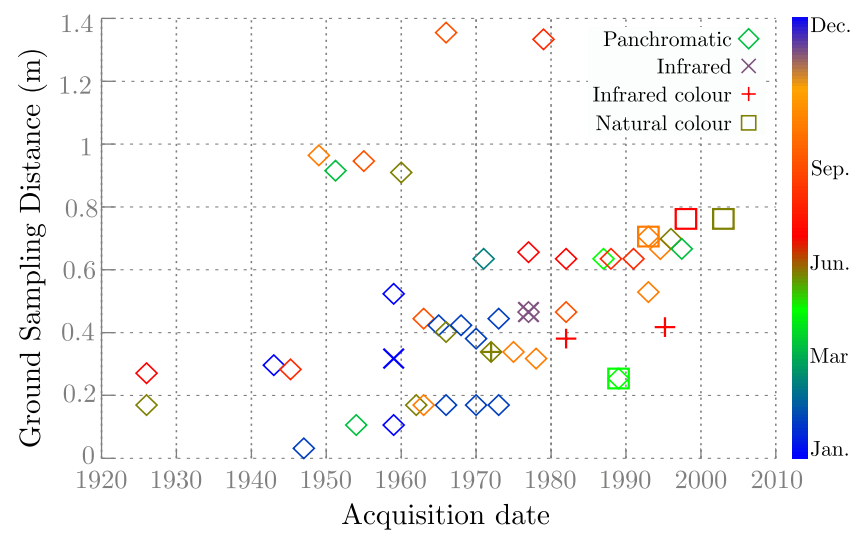

Fig. 1. Aerial surveys covering the study area depending on the Ground Sample Distance, the year and month of acquisition, and the type of images.

\section{B. Efficient photogrammetric workflows}

Photogrammetric softwares are perfectly adapted to process archival aerial image surveys [8], [9]. Major steps of a typical workflow that generates DSM and true orthoimages are described in the following. First, the interior orientation consists in establishing a mathematical relationship for each image between the camera frame coordinate and the image coordinate system (analog photo). This is done using the fiducial marks of each image. At the same time, tie points are extracted and matched on the input images. Then, relative image orientation is carried out using the tie points and the interior orientation. To produce the absolute image orientation in a cartographic system, coordinates of Ground Control Points (GCP) are needed. The DSM is later produced with a dense matching algorithm. In this workflow, true orthoimages are produced. As a result, the DSM computed is used to orthorectify each image of the aerial survey. At that step of the process, a radiometric equalization of the images can be performed (see below). Finally, the mosaicking step aims to merge each orthorectified image in a single orthoimage, covering the full area of interest.

5 epochs of the dataset, described in Table I, were selected in order to produce preliminary results. Residuals for the relative orientation step show that standard pipelines processing digital images can be applied. The orthoimages and DSM obtained for the study area are relevant and their planimetric accuracy, variable for each aerial survey, can be be roughly estimated to $2 \mathrm{~m}$ in average. Figure 3 shows these outputs on a restricted part of the study area.

\section{Challenges}

The challenges concerning fully automatic analysis of these aerial archival images deals with the ability to automatically (i) produce georeferenced orthoimages and Digital Surface Models, and (ii) to analyse the time series regarding its heterogeneity so as to generate land-cover or change maps.

\section{A. Automatic image registration}

In the process described in part II-B, tie point extraction and matching is a solved problem in photogrammetric computer vision, and can be automatically handled. Conversely, ground control points are usually manually selected in order to move from relative to absolute image orientation. Identifying permanent points over a significant period of time is a difficult and time consuming task [10]. Therefore, it should be automated. Two different approaches are conceivable either before or after orthoimage and DSM computation. Before photogrammetric processing, an automatic identification of permanent points within all image datasets could be carried out [11]. In this context, the addition of a current topographic database could be used to identify permanent structures, such as main road axis [12], or heritage buildings (e.g., churches or cemeteries), assuming the ground surface has not significantly be shifted. After photogrammetric processing, rigid image registration between the relative archival orthoimages/DSM, produced for every epoch, and the most recent orthoimage/DSM given in a cartographic projection should be performed. In that case, two steps may be considered. First, coarse absolute image orientation can be obtained using metadata of the localisation of the images (provided with raw images). Then, fine image registration techniques can be carried out. Because of the limited spectral resolution for some periods, the variation of the spatial resolution, and the fact that the observed territory changes in-between, methods taking into account spatial context [13] or fed with 3D information correspondence [14] would be particularly relevant.

\section{B. Radiometry of the orthoimages}

Issues are related to (i) the equalization of the radiometry of the set of images of a survey, and (ii) the heterogeneity of the radiometry in the time series. Equalization is often embedded in dense matching solutions: radiometric differences are minimised during the mosaicking process. However, there are very few implemented models for archival images, and differences can still be observed (Figure 2). Other errors stem from the workflow itself, when true images are produced: occlusions can be observed, the geometry of the objects can be corrupted if the DSM is used instead of a Digital Terrain Model [15]. Another issue is the temporal heterogeneity of the time series. Figure 1 shows data may have been acquired at different periods of the year, with varying spectral channels and cameras with different radiometric calibration set-ups. Figure 3 shows the orthoimages and the DSM generated for 5 dates of interest. It enhances the fact that no direct radiometric comparison can 
be performed. Vignetting correction, in addition to statistical equalization, has to be carried out [16]. Consequences of the radiometric issues on the ability to automatically analyse the images has to be assessed. Yet, it might not be compulsory to perform an accurate equalization as, for archival aerial images, most relevant features are probably linked to the geometric shapes rather than their radiometry. The hypothesis has to be validated but first land-cover classifications tend to enhance such conclusion (see below).

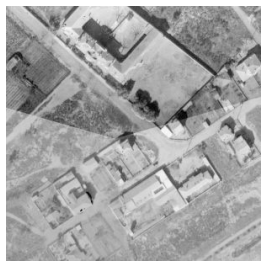

(a) Equalization

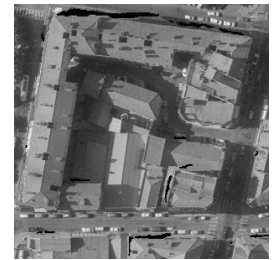

(b) Occlusions

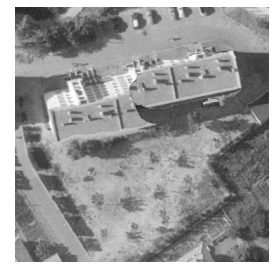

(c) Orthorectification
Fig. 2. Radiometric issues that can occur with true orthoimage generation from archival aerial images.

\section{How to classify images?}

Two major challenges can be identified performing landcover classifications for each date of the time series for a given nomenclature: the choice of the learning method, and the availability of a training set for each date. Here, we assume that state-of-the-art classifiers exhibit similar performance, which therefore mainly rely on the feature set. To illustrate the following challenges, a common classification pipeline was applied on the study area for a 5-class land-cover nomenclature \{buildings, bare soil, crops, high vegetation, low vegetation\}. Ground truth was acquired by visual analysis either on each date of the time series $\left(G T_{1}\right.$ in Table I), or on a 2016 airborne orthoimage $\left(G T_{2}\right.$ in Table I). Two sets of features were produced. The Ort features consist of the radiometry of the panchromatic channel and 14 texture parameters (Harralick+Structural Feature Set), derived from the true orthoimages. The DSM features are made of the elevation given by the DSM, along with a height-above-ground feature (obtained by morphological top-hat filtering of the DSM). $50 \%$ of the training samples were used to train a Random Forest classifier, and $50 \%$ to compute an average F-score (Table I). The landcover classifications obtained with the ground truth acquired on each date are provided in Figure 3.

First, one can see in Table I and Figure 3 that the use of all the features combined with ground truth produced for each date gives satisfactory preliminary results. 1966 and 1989 images acquired at very high spatial resolution lead to an average Fscore of $90 \%$, whereas lower score are observed for coarser spatial resolutions (1978 and 1995). Indeed, in addition to poor spectral resolution and the radiometric issues observed above, Figure 1 shows the variability of the spatial resolution between $0.1 \mathrm{~m}$ to $1 \mathrm{~m}$. Features derived from the DSM were found to be highly relevant. Adding them considerably improves the average F-score (Table I) and the final land-cover maps (Figure 3), especially for aerial surveys affected by radiometric issues (1954 and 1978). Generally speaking, the higher spatial resolution of the images the more complementary information is brought by DSM features. As a result, developing relevant and comparable features between the dates may be difficult. Deep learning methods using current land cover databases and textural information from high spatial resolution could then be inserted [17].

Besides the choice of the learning algorithm, the major challenge for classification is to retrieve discriminative training set for each image. Table I proves the inadequacy to use a single training set from the most recent periods. Even with the two sets of features, the average F-scores obtained are rather low, and decrease as images gets away from the training set. Domain adaptation techniques [18] should be envisaged in order to avoid defining training sets for each epoch [19]. This would also allow to take into account existing topographic databases for the learning step.

TABLE I. DESCRIPTION OF THE 5 DATASETS (1), PRECISION OF THE RELATIVE ORIENTATION (2) AND AVERAGE F-SCORE FOR DIFFERENT FEATURES AND TRAINING SET CONFIGURATIONS (3) (4)

\begin{tabular}{c|c|ccccc}
\hline & & $\mathbf{1 9 5 4}$ & $\mathbf{1 9 6 6}$ & $\mathbf{1 9 7 8}$ & $\mathbf{1 9 8 9}$ & $\mathbf{1 9 9 5}$ \\
\hline \multirow{3}{*}{$(1)$} & Acquisition scale & $1: 5,000$ & $1: 8,000$ & $1: 15,000$ & $1: 12,000$ & $1: 20,000$ \\
& Number of images & 19 & 15 & 10 & 12 & 10 \\
& GSD (m) & 0.11 & 0.17 & 0.32 & 0.26 & 0.42 \\
\hline \multirow{2}{*}{$(2)$} & Residuals (pix.) & 1.15 & 0.75 & 1.07 & 0.95 & 0.87 \\
\hline \multirow{2}{*}{$(3)$} & Ort \& GT & 53.11 & 72.86 & 75.36 & 76.65 & 79.80 \\
& Ort+DSM \& GT & 72.74 & 88.48 & 84.15 & 89.09 & 84.20 \\
\hline \multirow{2}{*}{$(4)$} & Ort \& GT & 40.01 & 35.82 & 36.27 & 41.85 & 54.70 \\
& Ort+DSM \& GT & 46.05 & 55.65 & 48.09 & 63.81 & 57.67 \\
\hline
\end{tabular}

\section{How to handle heteregenous times series?}

Assuming that land cover classification can be performed to each aerial survey independently, noisy label maps are likely to be obtained. In Figure 3, the less detailed scale of acquisition of the images decreases the quality of the DSM (1978 and 1995): building do not exhibit sharp contours. Noisy DSM due to poor radiometry can as well generate confusions between low and high vegetation (1954). The same problem can be observed if one is interested in the evolution of a specific feature such as the elevation computed from the DSM for instance. Ensuring a temporal consistency between variables (such as elevation) and land-cover maps appears to be mandatory.

The fact that time series of images are long and dense (Figure 1) offers two opportunities: (i) selection of the most appropriate epoch(s) for detecting a specific pattern (e.g., change); (ii) temporal regularisation of label maps [20]. In both cases, metadata concerning the aerial surveys and the precision of the relative image orientation should be taken into account. Methods employed for analysing archives of Landsat and SPOT data become highly relevant [21].

\section{CONCLUSION}

In this paper, opportunities and challenges to use archival aerial images have been identified. To illustrate challenges, a preliminary dataset over a urban area that faced significant changes has been produced. Future work will consist in handling such challenges. Producing the complete time series over three study areas and make them available to the community is another objective of this work. 


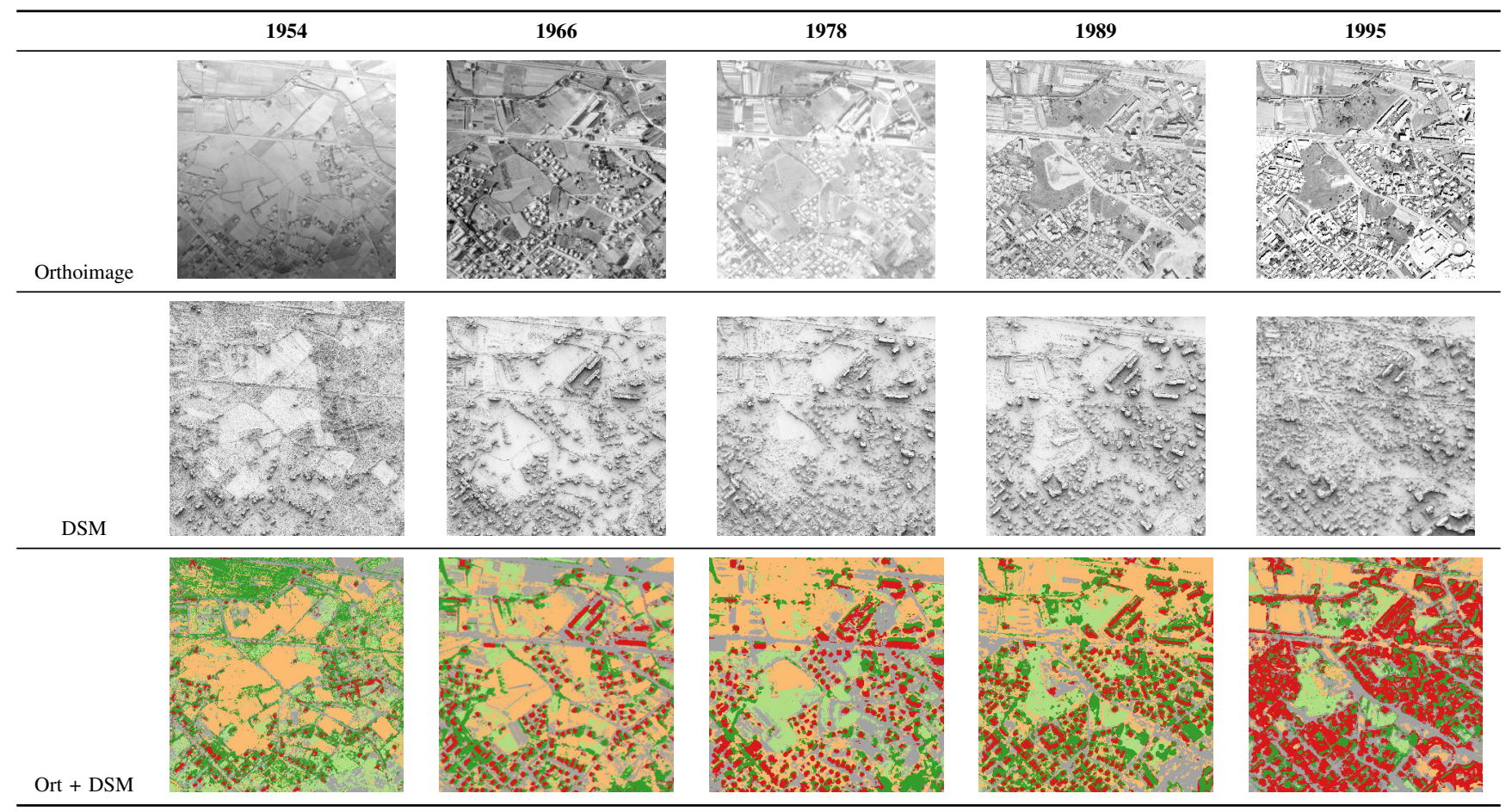

Buildings, Bare soil, Crops, High Vegetation, Low Vegetation

Fig. 3. True orthoimages, DSM, and land-cover maps, obtained using a training set produced by date, and features derived both from the orthoimages and the DSM (5-class nomenclature on a $800 \mathrm{~m} \times 800 \mathrm{~m}$ area of the study site).

\section{REFERENCES}

[1] X.-P. Song, J. O. Sexton, C. Huang, S. Channan, and J. R. Townshend, "Characterizing the magnitude, timing and duration of urban growth from time series of landsat-based estimates of impervious cover," Remote Sensing of Environment, vol. 175, pp. 1-13, 2016.

[2] M. Barthelemy, P. Bordin, H. Berestycki, and M. Gribaudi, "Selforganization versus top-down planning in the evolution of a city," Nature - Scientific reports, vol. 3, 2013.

[3] S. Leyk, R. Boesch, and R. Weibel, "Saliency and semantic processing: Extracting forest cover from historical topographic maps," Pattern Recognition, vol. 39, no. 5, pp. 953-968, 2006.

[4] P.-A. Herrault, D. Sheeren, M. Fauvel, and M. Paegelow, Geographic Information Science at the Heart of Europe. Springer, 2013, ch Automatic Extraction of Forests from Historical Maps Based on Unsupervised Classification in the CIELab Color Space, pp. 95-112.

[5] N. Micheletti, S. N. Lane, and J. H. Chandler, "Application of archival aerial photogrammetry to quantify climate forcing of alpine landscapes," The Photogrammetric Record, vol. 30, no. 150, pp. 143-165, 2015.

[6] USGS, "Aerial photo single frames. United States Geological Survey. https://lta.cr.usgs.gov/single-frame-records/,"accessed Apr. 12, 2016.

[7] IGN, "Géoportail. http://www.geoportail.gouv.fr/accueil/,"accessed Sep. 2, 2016.

[8] M. Pierrot-Deseilligny and I. Clery, "Apero, an open source bundle adjusment software for automatic calibration and orientation of set of images," in 3DARCH, 2011.

[9] S. Nebiker, N. Lack, and M. Deuber, "Building change detection from historical aerial photographs using dense image matching and objectbased image analysis," Remote Sensing, vol. 6, no. 9, pp. 8310-8336, 2014.

[10] I. Korpela, "Geometrically Accurate Time Series of Archived Aerial Images and Airborne Lidar Data in a Forest Environment," Silva Fennica, vol. 40, no. 1, pp. 109-126, 2006.
[11] S. Nagarajan and T. Schenk, "Feature-based registration of historical aerial images by area minimization," ISPRS Journal of Photogrammetry and Remote Sensing, vol. 116, pp. 15-23, 2016.

[12] I. Cléry, M. Pierrot-Deseilligny, and B. Vallet, "Automatic georeferencing of a heritage of old analog aerial photographs," Photogrammetric Computer Vision, vol. 2, no. 3, p. 33, 2014.

[13] J. Woo, M. Stone, and J. L. Prince, "Multimodal registration via mutual information incorporating geometric and spatial context," IEEE TIP, vol. 24, no. 2, pp. 757-769, 2015.

[14] O. C. Ozcanli, Y. Dong, J. L. Mundy, H. Webb, R. Hammoud, and V. Tom, "Automatic geolocation correction of satellite imagery," IJCV, vol. 116, no. 3, pp. 263-277, 2016.

[15] R. Ma, "Rational function model in processing historical aerial photographs," Photogrammetric Engineering \& Remote Sensing, vol. 79, no. 4, pp. 337-345, 2013.

[16] S. J. Kim and M. Pollefeys, "Robust radiometric calibration and vignetting correction,” IEEE TPAMI, vol. 30, no. 4, pp. 562-576, 2008.

[17] A. Lefebvre, T. Corpetti, and L. H. Moy, "Estimation of the orientation of textured patterns via wavelet analysis," Pattern Recognition Letters, vol. 32, no. 2, pp. 190-196, 2011.

[18] W. Li, L. Duan, D. Xu, and I. W. Tsang, "Learning with augmented features for supervised and semi-supervised heterogeneous domain adaptation," IEEE TPAMI, vol. 36, no. 6, pp. 1134-1148, 2014.

[19] D. Tuia, C. Persello, L. Bruzzone, "Domain Adaptation for the Classification of Remote Sensing Data: An Overview of Recent Advances," IEEE GRSM, vol. 4, no. 2, pp. 41-57, 2016.

[20] S. P. Abercrombie and M. A. Friedl, "Improving the consistency of multitemporal land cover maps using a Hidden Markov Model," IEEE TGRS, vol. 54, no. 2, pp. 703-713, 2016.

[21] E. Hamunyela, J. Verbesselt and M. Herold, "Using spatial context to improve early detection of deforestation from Landsat time series," Remote Sensing of Environment, vol. 172, pp. 126-138, 2016. 